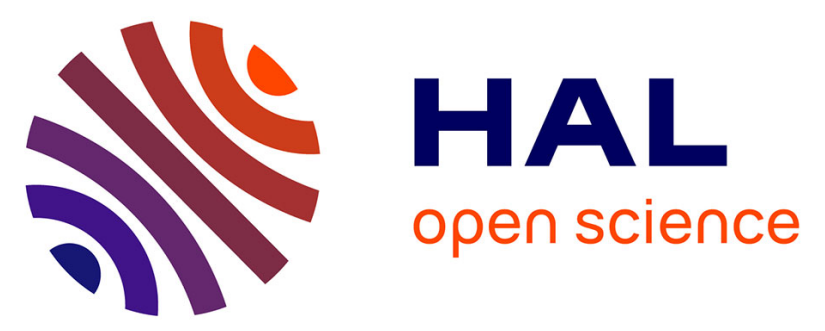

\title{
Application of Multi-Objective Genetic Algorithms to Two Case Studies of Reliability Efficiency Analysis and Optimal Expansion of Electrical Transmission Networks
}

\author{
F. Cadini, Enrico Zio, L. R. Golea, C. A. Petrescu
}

\section{- To cite this version:}

F. Cadini, Enrico Zio, L. R. Golea, C. A. Petrescu. Application of Multi-Objective Genetic Algorithms to Two Case Studies of Reliability Efficiency Analysis and Optimal Expansion of Electrical Transmission Networks. Proceedings of the Institution of Mechanical Engineers, Part O: Journal of Risk and Reliability, 2011, 225 (3), pp.365-374. 10.1177/1748006XJRR320 . hal-00609657

\section{HAL Id: hal-00609657}

https://hal-centralesupelec.archives-ouvertes.fr/hal-00609657

Submitted on 26 Jul 2012

HAL is a multi-disciplinary open access archive for the deposit and dissemination of scientific research documents, whether they are published or not. The documents may come from teaching and research institutions in France or abroad, or from public or private research centers.
L'archive ouverte pluridisciplinaire HAL, est destinée au dépôt et à la diffusion de documents scientifiques de niveau recherche, publiés ou non, émanant des établissements d'enseignement et de recherche français ou étrangers, des laboratoires publics ou privés. 


\title{
APPLICATION OF MULTI-OBJECTIVE GENETIC ALGORITHMS TO TWO CASE STUDIES OF RELIABILITY EFFICIENCY ANALYSIS AND OPTIMAL EXPANSION OF ELECTRICAL TRANSMISSION NETWORKS
}

\author{
F. Cadini ${ }^{1}$, E. Zio ${ }^{1,2}$, L.R. Golea ${ }^{1}$, C.A. Petrescu ${ }^{1}$ \\ ${ }^{1}$ Politecnico di Milano, Milan, Italy \\ ${ }^{2}$ Ecole Centrale Paris- Supelec, Paris, France
}

\begin{abstract}
Two applications of multi-objective genetic algorithms to the analysis and optimization of electrical transmission networks are reported to show the potential of these combinatorial optimization schemes in the treatment of highly interconnected, complex systems. In a first case study, an analysis of the topological structure of an electrical power transmission system of literature is carried out to identify the most important groups of elements of different sizes in the network. The importance is quantified in terms of group closeness centrality. In the second case study, an optimization method is developed for identifying strategies of expansion of an electrical transmission network by addition of new lines of connection which are optimally identified with respect to the objectives of improving the transmission reliability, while maintaining the investment cost limited.
\end{abstract}

Keywords: critical infrastructures, reliability efficiency, group closeness centrality measure, multi-objective genetic algorithms. 


\section{INTRODUCTION}

Society is heavily dependent on many technological, natural and even social complex systems which are hierarchies of networks of components (also called nodes, vertices or elements) interacting through links (also called edges, arcs or connections). The apparent ubiquity of networks leads to a fascinating set of problems common to biological, ecological, technological and social complex systems, regarding how the underlying network topology influences the system behavior and its robustness to faults and attacks. In this regard, the identification and quantification of their vulnerabilities, i.e. the set of flaws and weaknesses in the design, implementation, operation, and/or management of an infrastructure system or its elements that renders them susceptible to destruction or incapacitation when exposed to a hazard or threat, becomes crucial for designing the adequate protections, mitigation and emergency actions against their failures [1-3].

Given the complexity of these highly distributed and interconnected infrastructures, performing a systematic analysis of their vulnerability and robustness to failure becomes difficult if one resorts only to traditional probabilistic safety assessment (PSA) methods, so that new complementary approaches of network analysis are emerging for characterizing the network resistance to failure and identifying its most vulnerable elements [4-10]. To this aim, indicators of both the global reliability of the network and of the centrality of its elements have been introduced [11]. In particular, the global reliability efficiency indicators measure how well connected are the nodes of the network accounting not only for the topology of the network, but also for the failure probabilities of all the connections; this indicator differs from the classical reliability definition in that it represents general properties of the system and it is not defined with respect to a specific system task.

On the other hand, various measures of importance of the network elements can be defined, which capture different structural aspects of the interconnection paths among network nodes. Recently, these measures have been extended to rank the importance of groups of elements for taking into 
account the potential synergies between elements whose individual centrality may not be so relevant and dealing with the risk associated to changes in network technical specifications which typically impact groups of components [11].

Another direction of improvement in the definition of network importance measures regards the representation of the actual physical behavior of the system, e.g. in terms of the electric power or hydraulic flow in the network, etc [12].

In this paper, two applications of multi-objective genetic algorithms (MOGAs) are reported with regards to the analysis and optimization of electrical transmission networks. In the first case study, the electrical transmission network system of the IEEE (Institute of Electrical and Electronic Engineers) 14 BUS (a portion of the American Electric Power System) is considered [13] and a MOGA is used to solve an optimization problem in which the decision variables identify groups of components and the objectives are to maximize the importance of the groups while minimizing their dimension. This formulation guides the MOGA search towards the identification of the most important single components, couples of components, triplets, and so forth in the topological structure of the network.

In the second case study, a MOGA is developed for identifying strategies of expansion of an electrical network in terms of new lines of connection to add for improving the reliability of its transmission service, while maintaining limited the investment cost. The typical large size of electrical networks offers a combinatorial number of potential solutions of new connections, so that classical optimization techniques become inapplicable. For this reason, we resorted to a MOGA driven by the objectives of maximizing the network global reliability efficiency [14] and minimizing the cost of the added connections. To realistically restrict the search space to small numbers of new connections the so-called guided multi-objective genetic algorithm (G-MOGA) has been applied. In this approach, the search is based on the guided domination principle which allows to change the shape of the dominance region specifying maximal and minimal trade-offs between the different objectives so as to efficiently guide the MOGA towards Pareto-optimal solutions 
within these boundaries [15]. The performance of this search approach is tested on a case study based on the IEEE (Institute of Electrical and Electronic Engineers) RTS (Reliability Test System) $96[16]$.

The paper is organized as follows. Section 2 presents the group closeness centrality measure which can be used to quantify the importance of groups of nodes. The concept of network global reliability efficiency is also presented. A short introduction to the basic concepts behind the optimization procedure by Multi-Objective Genetic Algorithms is given in Section 3. In Section 4 and Section 5, the case studies regarding the IEEE 14 BUS network system and IEEE RTS 96 are presented and solved by MOGA. Conclusions on the outcomes of the analysis are eventually drawn in Section 6.

\section{TOPOLOGICAL GROUP CLOSENESS CENTRALITY AND GLOBAL RELIABILITY EFFICIENCY}

Mathematically, the topological structure of a network can be represented as a graph $\mathrm{G}(N, K)$ with $N$ nodes connected by $K$ edges. The connections are defined in an $N \times N$ adjacency matrix $\left\{a_{i j}\right\}$ whose entries are 1 if there is an edge joining node $i$ to node $j$ and 0 otherwise.

Depending on the specific definition, a centrality measure describes the way in which a node interacts/communicates with the rest of the network, thus providing a way of prioritization of the importance of the nodes for network communication. To evaluate the role played by a group of nodes with respect to its connectivity, the group closeness centrality [17], $\mathrm{C}^{\mathrm{C}}(\mathrm{g})$, is considered; this measure is based on the idea that a node can quickly interact with all other nodes if it is easily accessible by (close to) all others. If $d_{i j}$ is the topological shortest path length between nodes $i$ and $j$ (i.e., the minimum number of arcs on a path connecting them), the closeness of a group $\mathrm{g}$ of $N_{\mathrm{g}}$ nodes is the inverse of the node's mean distance from the members of the group to all vertices outside the group: 


$$
\mathrm{C}^{\mathrm{C}}(\mathrm{g})=\frac{N-N_{\mathrm{g}}}{\sum_{i \in N_{\mathrm{g}}, j \in N} d_{i j}}
$$

This measure is normalized by dividing the distance score into the number of non-group members, with the result that larger numbers indicate greater centrality.

When the group consists of a single node, the group closeness centrality is the same as the individual node closeness centrality [6], [18-19].

To capture the failure behavior of the network, the reliability of its connecting edges is included in the framework of analysis by means of the formalism of weighted networks, the weight $w_{i j}$ associated to the edge between the pair of nodes $i$ and $j$ being its reliability:

$$
p_{i j}=e^{-\lambda_{i j} \cdot T}
$$

where $\lambda_{i j}$ is the failure rate of edge $i j$ linking nodes $i$ and $j$ and $T$ is a reference time ( $T=1$ year, in this work).

On the basis of the adjacency and reliability matrices $\left\{a_{i j}\right\}$ and $\left\{p_{i j}\right\}$ (or the complementary failure probability matrix $\left\{q_{i j}\right\}$ ), the matrix of the most reliable path lengths $\left\{r d_{i j}\right\}$ can be computed [14]:

$$
r d_{i j}=\min _{\gamma_{i j}}\left(\frac{1}{\prod_{m n \in \gamma_{i j}} p_{m n}}\right)=\min _{\gamma_{i j}}\left(\frac{1}{\prod_{m n \in \gamma_{i j}}\left(1-q_{m n}\right)}\right)
$$

where the minimization is done with respect to all paths $\gamma_{i j}$ linking nodes $i$ and $j$ and the product extends to all the edges of each of these paths. Note that $1 \leq r d_{i j} \leq \infty$, the lower value corresponding 
to the existence of a perfectly reliable path connecting $i$ and $j$ (i.e. $p_{m n}=1, q_{m n}=0 \forall m n \in i j$ ) and the upper value corresponding to the situation of no paths connecting $i$ and $j$ (i.e. $p_{m n}=0, q_{m n}=1$ ).

The group reliability closeness centrality can then be computed as in equation 1 , with $r d_{i j}$ replacing $d_{i j}$

The global reliability efficiency $\mathrm{RE}[\mathrm{G}]$ of the graph $\mathrm{G}$ can also be defined as [14]:

$$
\mathrm{RE}[\mathrm{G}]=\frac{1}{N(N-1)} \sum_{i, j \in \mathrm{G}, i \neq j}\left(1 / r d_{i j}\right)
$$

In practical terms, the global reliability efficiency indicates how well connected are the nodes of the network taking into account their connection failure probability. For example, the design of a network with very few but highly reliable connections between the nodes is preferable with respect to a densely connected design with connections characterized by large failure rates.

\section{MULTI-OBJECTIVE GENETIC ALGORITHMS}

Genetic Algorithms (GAs) are optimization methods aiming at finding the global optimum of a set of real objective functions, $\mathrm{F} \equiv\{\mathrm{f}(\cdot)\}$, of one or more decision variables, $U \equiv\{u\}$, possibly subject to various linear or non linear constraints. The terminology adopted in GAs contains many terms borrowed from biology, suitably redefined to fit the algorithmic context. Thus, GAs operate on a set of (artificial) chromosomes, which are strings of numbers, generally sequences of binary digits (bits) 0 and 1, coding the values of the decision variables. The values of the objective functions in correspondence of the values of the decision variables of a chromosome, give the fitness of that chromosome. The GA search is performed by constructing a sequence of populations of chromosomes, the individuals of each population being the children of those of the previous population and the parents of those of the successive population. The initial population is generated 
by randomly sampling the bits of all the strings; at each step in the search sequence, the new population is obtained by probabilistically manipulating the strings of the old population with fitness-improving rules which mimic genetic evolution. The search sequence continues until a preestablished optimality termination criterion is reached.

Typically, several possibly conflicting objective functions $\mathrm{f}_{i}(\cdot), i=1,2, \ldots, n_{f}$, must be evaluated in correspondence of each decision variable vector $U$ in the search space. In this case, the GA search proceeds by comparing the solutions in terms of the concepts of Pareto optimality and dominance [20]: with reference to a maximization problem proposed, a solution $U_{a}$ is said to dominate $U_{b}$ if

$$
\begin{gathered}
\forall i \in\left\{1,2, \ldots, n_{f}\right\}, \mathrm{f}_{i}\left(U_{a}\right) \geq \mathrm{f}_{i}\left(U_{b}\right) \\
\text { and } \\
\exists j \in\left\{1,2, \ldots, n_{f}\right\}, \mathrm{f}_{j}\left(U_{a}\right)>\mathrm{f}_{j}\left(U_{b}\right) .
\end{gathered}
$$

In all other cases of decision variable vectors which are not dominated by any other of a given set, including when $\forall l \in\left\{1,2, \ldots, n_{f}\right\}, \mathrm{f}_{l}\left(U_{a}\right)=\mathrm{f}_{l}\left(U_{b}\right), U_{a}$ and $U_{b}$ are nondominated with respect to this set within the entire search space, are said to be Pareto efficient and constitute the so called Pareto optimal front or Pareto set, which is the object of the optimization. From an engineering point of view, restricting the attention to the set of choices that are Pareto efficient allows a designer to make tradeoffs within this set, rather than taking into account the full range of every decision variable. 


\section{CASE STUDY 1}

\subsection{IEEE 14 BUS electrical transmission network}

The topological structure of the electrical transmission network system of the IEEE 14 BUS is considered for the analysis of the importance of groups of components, measured in terms of reliability closeness centrality. The system considered represents a portion of the American Electric Power System and consists of 14 bus locations connected by 20 lines and transformers. The topology of the system can be represented by the graph $\mathrm{G}(14,20)$ of Figure 1 .

Figure 1. Graph representation of the IEEE 14 BUS transmission network

\subsection{Identification of most central groups of nodes by MOGA}

Let the generic centrality measure of a group of nodes be indicated as:

$$
\mathrm{C}\left(x_{1}, x_{2}, \ldots, x_{N}\right)
$$

with $x_{i}=1$ if $i$ belongs to the group, $x_{i}=0$ otherwise. For example, in a network with $N=3$ components, $C(1,0,0)$ indicates the centrality of the first node alone; $C(0,0,1)$ indicates the centrality of the third node alone; $\mathrm{C}(1,0,1)$ indicates the centrality of the pair made of the first and third nodes.

In a network with $N$ nodes, the number of groups (single nodes, pairs, triplets, and so forth) that in principle can be formed is $2^{N}$. A complete centrality analysis of all groups to find the most central is therefore impractical for large networks.

To overcome this obstacle, the task of determining the most central groups of components can be framed as an optimization problem with respect to the two following objectives:

$$
\begin{array}{cc}
\underset{\mathbf{x}}{\operatorname{Max}} \quad \mathrm{f}_{1}(\mathbf{x})=\mathrm{C}\left(x_{1}, x_{2}, \ldots, x_{N}\right) \\
\underset{\mathbf{x}}{\operatorname{Min}} \mathrm{f}_{2}(\mathbf{x})=\sum_{i=1}^{N} x_{i}
\end{array}
$$


with $x_{i}=1$ if $i$ belongs to the group, $x_{i}=0$ otherwise.

The need for introducing the second objective is due to the fact that inevitably the more nodes in a group, the higher its centrality and impact on the network; hence, a search directed only by the centrality of the groups (objective $f_{1}(\mathbf{x})$ ), with no control on their sizes (objective $f_{2}(\mathbf{x})$ ) would favor large groups, whose simultaneous failure or attack is however not likely. By introducing the second objective, which favors small groups, the search is guided to finding the most central groups preferably of small size, i.e., the single nodes, pairs, triplets and so forth.

A Genetic Algorithm (GA) can be devised to address the above optimization task. In the most typical applications of GAs, every proposal of solution, represented by the vector $\mathbf{x}$ of the independent decision variables (control parameters), is coded in a so-called chromosome, constituted by so-called genes, each one coding one decision variable of $\mathbf{x}$; a binary coding is widely used. Differently from these typical applications, the decision variables $\left(\mathrm{x}_{1}, x_{2}, \ldots, x_{N}\right)$ of the optimization problem considered in this paper are already in the form of a binary string. Therefore, single-gene chromosomes are used, directly coding the composition of the group. The dimension of the chromosome is equal to the number of nodes in the network. Table 1 shows two examples of chromosome coding for a system of 14 nodes.

Table 1. Examples of chromosomes coding groups of nodes in a network of 14 nodes

\section{Chromosome coding (14 bits)}

\begin{tabular}{cccccccccccccc}
\hline 1 & 2 & 3 & 4 & 5 & 6 & 7 & 8 & 9 & 10 & 11 & 12 & 13 & 14 \\
0 & 0 & 0 & 1 & 0 & 1 & 0 & 0 & 1 & 0 & 0 & 0 & 0 & 0 \\
1 & 0 & 0 & 0 & 0 & 1 & 0 & 1 & 1 & 0 & 0 & 0 & 1 & 0 \\
\hline
\end{tabular}

Corresponding nodes in the group

$4,6,9$

$1,6,8,9,13$

There are two kinds of operations performed in the genetic algorithms search for optimal solutions: i) genetic operations, that mimic the process of heredity of genes to create new offsprings (i.e., new solutions) at each generation and ii) evolution operations, which mimic the process of Darwinian 
evolution to create populations from generation to generation. The two basic types of genetic operators, i.e., crossover and mutation, provide the basic search mechanism of the GA and depend on the chromosome representation used. Crossover takes two individuals (i.e., two solutions) and produces two new individuals while mutation alters one individual to produce a single new solution. To use a GA for Multiobjective Optimization (MO) entails comparing two solutions with respect to the multiple objectives considered [21], [22]. In the case of a single-objective, the comparison is trivial: a vector solution $\mathbf{x}$ is better than another one, say $\mathbf{y}$, if the corresponding objective function (fitness) value $\mathrm{f}(\mathbf{x})$ is greater than $\mathrm{f}(\mathbf{y})$. A multiobjective optimization problem, instead, deals with $n_{f}$ objective functions $\mathrm{f}_{i}(\cdot), i=1,2, \ldots, n_{f}$; this requires that two solutions $\mathbf{x}$ and $\mathbf{y}$ are compared in terms of dominance of one solution over the other with respect to all $n_{f}$ objectives [23]. The multiobjective optimality search process, converges on a Pareto-optimal set of nondominated solutions, which provides a spectrum of possible choices for the decision-maker to a posteriori identify his or her preferred solution.

Table 2 summarizes the details of the implementation of the MOGA operators described in Section 3 along with a number of parameters that control the operation of the genetic algorithm such as the population size (i.e., the size of the evolving set of candidate solutions) and the number of generations (i.e., the duration of the evolution process). The optimization runs were performed starting from an initial random population.

Table 2. MOGA parameters and rules

\begin{tabular}{ll}
\hline Number of chromosomes & 200 \\
Number of generations & 200 \\
Selection & Fit-Fit \\
Replacement & Children-parents \\
Mutation probability & 0.001 \\
$\begin{array}{l}\text { Crossover probability } \\
\text { Number of generations }\end{array}$ & 1 \\
$\begin{array}{l}\text { without elitist selection } \\
\text { Fraction of parents chosen } \\
\text { with elitist selection }\end{array}$ & 0.25 \\
\hline
\end{tabular}


Figure 2 shows the results obtained on the importance of the group in terms of reliability closeness centrality. In the Figure, the values of the objective functions in correspondence of all the nondominated groups of nodes contained in the MOGA archive at convergence are shown to identify the two-dimensional Pareto-optimal surface (circles). The results are compared for validation with those obtained by exhaustive computation of all groups of nodes, i.e., the computation of the group reliability closeness centrality measure for all the possible combinations of $n$ out of $N$ nodes; due to the fact that the number of groups obtained is $2^{N}$, its implementation is feasible here thanks to the small size of the network but would require impractical computational resources for large networks.

Figure 2. Results of the multi-objective search of the most central groups of nodes in terms of reliability closeness centrality

Actually, different groups of equal size can have the same centrality measure value: Table 3 reports all the nondominated solutions contained in the archive, identified by the MOGA. For the groups composed of one, three and five nodes, there is only one combination that maximizes the reliability closeness centrality measure; on the other hand, there is more than one solution for the groups with sizes two, four and six to ten; this allows choosing the solution most suitable for a given purpose, e.g. system upgrade by components renewal, replacement, or redundancy allocation in order to use effectively the budget available.

In the present case, the smallest group with maximal reliability closeness is of size 10 and there are 2 of these. The group $\{1,2,3,5,7,10,11,12,13,14\}$ is particularly interesting because it does not contain the highly central node $\{4\}$ and contains the node $\{1\}$ that have the smallest individual reliability closeness centrality measure, as it can be seen in Table 4.

Table 3. Pareto optimal results of the multi-objective search for reliability closeness centrality groups 


\begin{tabular}{|c|c|c|}
\hline $\begin{array}{l}\text { Group } \\
\text { reliability } \\
\text { closeness } \\
\text { centrality }\end{array}$ & $\begin{array}{c}\text { Group } \\
\text { Size }\end{array}$ & Components \\
\hline 0.303 & 1 & 4 \\
\hline 0.47 & 2 & $(4,6),(6,9)$ \\
\hline 0.562 & 3 & $(2,6,9)$ \\
\hline 0.602 & 4 & $(1,2,6,9),(1,3,6,9),(2,3,6,9)$ \\
\hline 0.659 & 5 & $(1,2,3,6,9)$ \\
\hline 0.688 & 6 & $(1,2,3,6,7,9),(1,2,3,6,8,9)$ \\
\hline 0.761 & 7 & $\begin{array}{c}(1,2,3,5,7,10,13),(1,2,3,5,7,11,13),(1,2,3,6 \\
7,10,13),(1,2,3,6,7,10,14),(1,2,3,6,7,11,13) \\
(1,2,3,6,7,11,14)\end{array}$ \\
\hline 0.802 & 8 & $\begin{array}{c}(1,2,3,5,7,10,11,13),(1,2,3,5,7,10,12,13),(1, \\
2,3,5,7,11,12,14),(1,2,3,6,7,10,11,13),(1,2,3, \\
\quad 6,7,10,12,13),(1,2,3,6,7,11,12,14) \ldots\end{array}$ \\
\hline 0.868 & 9 & $\begin{array}{c}(1,2,3,5,7,10,11,12,13),(1,2,3,5,7,10,11,13 \\
14),(1,2,3,6,7,11,12,13,14) \ldots\end{array}$ \\
\hline 0.99 & 10 & $\begin{array}{c}(1,2,3,5,7,10,11,12,13,14),(1,2,3,6,7,10,11 \\
12,13,14)\end{array}$ \\
\hline
\end{tabular}

Table 4. Individual reliability closeness centrality

\begin{tabular}{cc}
\hline Node & $\begin{array}{c}\text { Reliability closeness } \\
\text { centrality }\end{array}$ \\
\hline 4 & 0.3031 \\
9 & 0.2998 \\
5 & 0.2835 \\
7 & 0.2742 \\
6 & 0.2716 \\
14 & 0.253 \\
10 & 0.2448 \\
13 & 0.2448 \\
11 & 0.2371 \\
2 & 0.2272 \\
8 & 0.2184 \\
12 & 0.2081 \\
3 & 0.1793 \\
1 & 0.1723 \\
\hline
\end{tabular}

To gain an engineering feeling of the impact on the network transmission performance resulting from the failure of the nodes of a group of a given reliability closeness centrality value, the relative global reliability efficiency variation of the network is reported in Figure 3. The relative variation of the global reliability efficiency due to the removal of a group of nodes is computed as the difference between the values of the global reliability efficiency (Equation 4) of the network with all the nodes 
of the group (and all the incident arcs) removed and of the original network with no nodes removed, normalized to the latter.

Figure 3. Relative variation of the global reliability efficiency as a function of group closeness centrality measure

The results show that there is a strong relation between the size of the groups removed and the efficiency of the network and that the most reliability-close central groups (i.e., those in the Pareto front) are indeed the most important also from the point of view of the negative impact on the network global reliability efficiency, when they fail.

\section{CASE STUDY 2}

\subsection{IEEE RTS 96 electrical transmission network}

The transmission network system IEEE RTS 96 (Figure 4) [16] consists of 24 bus locations (numbered in bold in the Figure) connected by 34 lines and transformers. The transmission lines operate at two different voltage levels, $138 \mathrm{kV}$ and $230 \mathrm{kV}$. The $230 \mathrm{kV}$ system is the top part of Figure 4, with 230/138 kV tie stations at Buses 11, 12 and 24.

Figure 4. IEEE RTS 96 transmission network

Figure 5 gives the representation of the graph $\mathrm{G}(24,34)$ of the transmission network; the corresponding $24 \times 24$ adjacency matrix $\left\{a_{i j}\right\}$ has entry equal to 1 if there is a line or transformer between bus locations $i$ and $j$ and 0 otherwise.

Figure 5. The IEEE RTS 96 graph representation 


\subsection{Optimal network expansion by MOGA}

A MOGA has been constructed for identifying the best improvements in the connection of the network, aimed at increasing its global reliability efficiency in transmission at acceptable costs. The improvements are obtained by addition of new lines between nodes with no direct connection in the original network. Given the lack of geographical information on the nodes locations, for simplicity and with no loss of generality, three typologies of lines have been arbitrarily chosen as the minimum, the mean and the maximum values of the failure rates of the transmission lines taken from [16]:

$$
\begin{aligned}
& \lambda_{1}=0.2267 \text { outages } / \mathrm{yr} \\
& \lambda_{2}=0.3740 \text { outages } / \mathrm{yr} \\
& \lambda_{3}=0.5400 \text { outages } / \mathrm{yr}
\end{aligned}
$$

The addition of a new line requires an investment cost assumed inversely proportional to the failure rate. The network cost can be then defined as:

$$
\mathrm{C}[\mathrm{G}]=\sum_{i, j \in N, i \neq j}\left(1 / \lambda_{i j}\right)
$$

The reliability cost of the original IEEE RTS 96 is $C[G]=332.0120$ in arbitrary monetary units and the reliability efficiency is $\mathrm{RE}[\mathrm{G}]=0.2992$, which is a relatively high value representative of a globally reliable network.

The network global reliability efficiency and cost are adopted as objective functions in the MOGA optimization of the network improved structure.

From the algorithmic point of view, a proposal of improvement amounts to changing from 0 to 1 the values of the elements in the adjacency matrix corresponding to the added connections. The only physical restriction for adding direct new connections is that the connected nodes must be at 
the same voltage level $(138$ or $230 \mathrm{kV})$, otherwise the addition of a transformer would also be needed. From the genetic algorithm point of view, the generation of proposals of network improvements can be achieved by manipulating a population of chromosomes, each one with a number of bits equal to 214 which is double the number of zeros (i.e., the number of missing direct connections $i j$ ) in the upper triangular half of the symmetric adjacency matrix $\left\{a_{i j}\right\}$. The bits are dedicated to each missing direct connection $i j$ so as to code the three different available types of lines with failure rates $\lambda_{1}, \lambda_{2}$ and $\lambda_{3}$ : in other words, the bit-string (00) is used to code the absence of connection, (01) connection line with a $\lambda_{1}$-type line, (10) connection with a $\lambda_{2}$-type line and (11) connection with a $\lambda_{3}$-type line. The initial population of 200 individuals is created by uniformly sampling the binary bit values.

During the genetic search, each time a new chromosome is created, the corresponding matrices $\left\{a_{i j}\right\}$ and $\left\{p_{i j}\right\}$ are constructed to compute the values of the two objective functions, network global reliability efficiency and cost of the associated improved network.

Figure 6 shows the Pareto dominance front (squares) obtained by the MOGA at convergence after $10^{3}$ generations; the circle represents the original network with $R E[G]=0.2992$ and $\mathrm{C}[\mathrm{G}]=332.0120$, while the star represents the network fully connected by the most reliable transmission lines $\lambda_{1}=0.2267 \mathrm{occ} / \mathrm{yr}$, for which $\mathrm{RE}[\mathrm{G}]=0.57$ and $\mathrm{C}[\mathrm{G}]=804.1072$.

Figure 6. Pareto front reached by the MOGA

The optimality search is biased from the beginning (from the initial population) towards highly connected network solutions, because the string (00) has a probability of 0.25 whereas the probability of adding a connection of any one of the three available types (i.e., the probability of the strings $01,10,11)$ is 0.75 ; this drives the population evolution to highly connected networks in the Pareto front (squares in Figure 6), all with values $R E[G] \geq 0.4417, C[G] \geq 454.4738$ and numbers of added connections exceeding 60. 
In practical applications only a limited number of lines can be added, due to the large investment costs and other physical constraints. To drive the genetic search towards low cost solutions (i.e., low number of added lines) maximal and minimal trade-offs to the two objectives of the optimization (network global reliability efficiency and cost) can be defined within a Guided MultiObjective Genetic Algorithm (G-MOGA) scheme, [15]. The preferential optimization has been performed by using G-MOGA, with the same population size, evolution procedures and parameters of the previous search. In this approach, the search is guided by defining the maximal and minimal trade-offs that allow to identify a precise section of the Pareto front. The values of the trade-off parameters have been set by trial-and-error to $a_{12}=331.3157$ and $a_{21}=0$; the search converges to a small number of solutions in a Pareto front which is more concentrated on low cost networks, characterized by a limited number of added connections (asterisks in Figure 6).

Table 5 lists the five solutions of lowest cost identified by the G-MOGA search: the added connections improve the network global reliability efficiency and they do so with relatively small costs.

Table 5. The five solutions on the Pareto front obtained by the G-MOGA

\begin{tabular}{cc}
\hline \multicolumn{2}{c}{ G-MOGA } \\
\hline Reliability Efficiency & Cost \\
\hline 0.3072 & 337.6 \\
0.3168 & 339.4 \\
0.3186 & 339.4 \\
0.3187 & 339.4 \\
0.3193 & 339.4 \\
\hline
\end{tabular}

\section{CONCLUSIONS}

This paper has illustrated the use of MOGA in the analysis of complex, interconnected network systems.

First, the electrical transmission network system of the IEEE 14 BUS has been analyzed by MOGA to identify the importance of groups of components, measured in terms of their centrality in the structure of interconnection paths. The results obtained using the group reliability closeness 
centrality measure as importance indicator have shown that the groups classified as most central indeed contain the nodes of individual highest centrality but may also include nodes with a relatively low centrality. The groups most important from the point of view of group reliability closeness have been shown to be also the most vulnerable to direct attacks, by a crude procedure of removal of nodes and arcs one at a time.

Then, a MOGA for improving the electrical transmission network IEEE RTS 96 has been implemented with the objective of identifying the lines to be added for maximizing the network transmission reliability efficiency, while maintaining the investment costs limited. A preferential procedure of optimization has been performed by using G-MOGA for individuating realistic network expansion solutions made of few new transmission lines.

The original contributions of the paper lie in i) the innovative bit coding strategies within a MOGA approach; ii) the definition of the appropriate objective functions for efficiently handling the combinatorial optimization; iii) the adoption of the guided search, to realistically steer the optimization towards practically implementable solutions of network improvement; iv) the engineering analysis and implementation of the results of the otherwise "blind" MOGA automatic optimization. The case studies have been chosen purposely for the simplicity of the structure of the networks, which has allowed the verification of the proposed approach, which demonstrates the coherence of the MOGA searches with respect to the physical behavior of the two networks.

From the point of view of the physical analysis of the electrical transmission system which the networks represent, some limitations affect the results obtained. These limitations are all related to the fact that the analysis performed focuses only on the topological features of the network, thus neglecting its physical characteristics; this is not realistic for electrical transmission networks.

Work is currently undergoing in establishing effective ways of bringing these physical characteristics into the topological analysis.

\section{Acknowledgments:}


This work has been funded by the Foundation pour une Culture de Securite Industrielle of Toulouse, France, under the research contract AO2006-01.

\section{REFERENCES}

[1] CNIP'06 (March 2006). Proceedings of the International Workshop on Complex Network and Infrastructure Protection, Rome, Italy, 28-29.

[2] Rocco, C. M., Zio, E., and Salazar, D. E. (2007). Multiobjective evolutionary optimisation of the protection of complex networks exposed to terrorist hazard. Aven, T.; Vinnem, J. E. (edts.): Risk, Reliability and Societal Safety. Proceedings of the European Conference on Safety and Reliability (ESREL 2007) Stavanger, Norway, 25-27 June 2007, London: Taylor and Francis, vol. 1, pp. 899905.

[3] Vulnerability, Reliability and Safety of Complex Networks and Critical Infrastructures, Special Sessions I and II. Aven, T.; Vinnem, J. E. (edts.): Risk, Reliability and Societal Safety. Proceedings of the European Conference on Safety and Reliability (ESREL 2007) Stavanger, Norway 25-27 June 2007, London: Taylor and Francis, vol. 1.

[4] Cadini, F., Zio, E., and Petrescu, C. A. Using centrality measures to rank the importance of the components of a complex network infrastructure. Proceedings of the 3rd International Workshop on Critical Information Infrastructures Security (CRITIS'08), Ed. Springer, 13-15 October 2008, Frascati (Rome), Italy.

[5] Freeman, L.C. (1979). Centrality in Social Networks: Conceptual Clarification, Social Networks 1, pp. 215-239.

[6] Hines, H. and Blumsack, S. A centrality measure for electrical networks. Proceedings of the 41st Hawaii International Conference on System Science, IEEE Computer Society Press, pp.185, Waikoloa, Big Island, Hawaii, 07-10 January 2008. 
[7] Koonce A. M., Apostolakis G.E., Cook B.K. (2007). Bulk power risk analysis: Ranking infrastructure elements according to their risk significance, Int J Electr Power Energ Syst, doi:10.1016/j.ijepes.2007.06.013.

[8] Newmann, M. E. J. (2003). The structure in function of complex networks, SIAM Review 45:2, pp 167-256.

[9] Zio, E., Petrescu, C. A., and Sansavini, G. Vulnerability analysis of a power transmission system. Proceedings of the International Probabilistic Safety Assessment and Management Conference (PSAM'09), 18-23 May 2008, Edge Publication Group Limited, Hong Kong, China.

[10] Rocco, M.C., Ramírez-Márquez, J.E., Salazar, D.E., Hernández, I. (2010) Implementation of Multiple-Objective Optimization for Vulnerability Analysis of Complex Networks. Journal of Risk and Reliability, (to appear).

[11] Zio, E. Computational methods for reliability and risk analysis, in Quality, Reliability and Engineering Statistics series, vol. 14, 2009 (World Scientific, Singapore).

[12] Rocco, C., Ramirez-Marquez, J.E., Salazar, D. and Zio, E. (2010). A Flow Importance Measure with Application to an Italian Transmission Power System, International Journal of Performability Engineering, Vol. 6, No. 1, pp. 53-61.

[13] Christie, R. (1993). The IEEE 14 BUS data can be found on: http://www.ee.washington.edu/research/pstca/, University of Washington.

[14] Zio, E. (2007). From Complexity Science to Reliability Efficiency: A New Way of Looking at Complex Network Systems and Critical Infrastructures, Int. J. Critical Infrastructures, Vol. 3, Nos. 3/4, pp. 488-508.

[15] Zio E., Baraldi P., Pedroni N. (2009). Optimal power system generation scheduling by multiobjective genetic algorithms with preferences, Reliab. Eng. Sys. Safety, Vol. 94, Issue 2, pp. 432444.

[16] Billinton, R. and Li, W. Reliability assessment of electric power systems using Monte Carlo methods, 1994 (Plenum Publishing, New York). 
[17] Everett, M.G., Borgatti, S.P. (1999). The centrality of groups and classes, Journal of Mathematical Sociology, Vol. 23, N. 3, pp. 181-201.

[18] Sabidussi G. (1966). The Centrality Index of a Graph, Psychometrika, n.31.

[19] Wasserman S., Faust K. (1994). Social Networks Analysis, Cambridge U.P., Cambridge, UK.

[20] Goldberg D.E. (1989). Genetic algorithms in search, optimization, and machine learning, Addison-Wesley Publ. Co.

[21] Carlos, S., Martorell, S., Villanueva, J.F., Serradell, V. \& Sànchez, A. (2007). A multiobjective genetic algorithm for RAMS + C optimization with uncertain variables. Proceedings of the 2007 European Safety and Reliability Conference (ESREL 2007), Stavanger, Norway, 25-27 June, 115121.

[22] Toshinsky, V.G., Sekimoto H., and G.I. Toshinsky. (2000). A method to improve multiobjective genetic algorithm optimization of a self-fuel-providing LMFBR by niche induction among nondominated solutions. Annals of Nuclear Energy, 27: 397-410.

[23] Sawaragi, Y., Nakayama, H., Tanino, T. (1985). Theory of multiobjective optimization. Academic Press, Orlando, Florida. 